\title{
Presencia de los alelos DRD4/7R y DAT1/10R en miembros de familias chilenas con síndrome de déficit atencional con hiperactividad
}

\author{
Ximena Carrasco $\mathrm{Ch}^{\mathbf{1}, 3}$, Paula Rothhammer $\mathrm{A}^{\mathbf{1 , 2}}$, \\ Mauricio Moraga $\mathbf{V}^{\mathbf{1}}$, Hugo Henríquez $\mathrm{B}^{\mathbf{1}}$, \\ Francisco Aboitiz D ${ }^{2}$, Francisco Rothhammer $E^{1}$. \\ Presence of DRD4/7R and DAT1/10R \\ allele in Chilean family members \\ with attention deficit hyperactivity \\ disorder
}

Background: Genes for dopamine receptor DRD4 and dopamine transporter DAT1 have been implicated in attention deficit with hyperactivity disorder (ADHD). However, the findings are not conclusive. More studies in populations with different genetic brackgrounds may contribute to solve the discrepancies observed. Aim: To test the hypothesis that affected members of Chilean families exhibit higher frequencies of the DRD4/7R and DAT1/10R alleles then their healthy sibs. Material and Methods: The parents of 51 children belonging to families of the Metropolitan Region of Chile, were approached to obtain clinical histories and blood samples, after the signature of a written informed consent. ADHD was diagnosed according to DSM-IV criteria, and intellectual coefficient was tested using the WISC-R test. Genomic DNA was extracted from lymphocytes and amplified by PCR. Results: The 7R allele was identified in 13 out of 26 subjects diagnosed as $\mathrm{ADHD}$ and in 6 of 25 healthy sibs ( $\mathrm{p} \varangle 0.05$ ). Parents with a history of ADHD, were compared with their healthy counterparts, exhibiting an identical tendency, that did not reach statistical significance. No significant differences in the frequencies of DAT1/10R alleles, were observed between cases and controls or their parents. Conclusions: Our results showed that ADHD in Chilean families is associated with the presence of DRD4/7Rallele (Rev Méd Chile 2004; 132: 1047-52).

(Key Words: Attention deficit hyperactivity disorder; Dopamine-binding protein; Receptors, dopamine, DRD4)

Recibido el 5 de abril, 2004. Aceptado en versión corregida el 13 de julio, 2004.

${ }^{1}$ Instituto de Ciencias Biomédicas, Facultad de Medicina, Universidad de Chile. ${ }^{2}$ Departamento de Psiquiatría, Escuela de Medicina, Pontificia Universidad Católica de Chile. ${ }^{3}$ Servicio de Neurología y Psiquiatría Infantil, Hospital Luis Calvo Mackenna.

Correspondencia a: Prof. Dr. Francisco Rothhammer E. Programa de Genética Humana, Instituto de Ciencias Biomédicas, Facultad de Medicina, Universidad de Chile. Av Independencia 1027- Casilla 70061- Santiago 7 - Chile. E mail: frothham@med.uchile.cl 
$\mathrm{E}^{1}$ Síndrome de Déficit Atencional (SDA) constituye una de las condiciones neuropsiquiátricas de la infancia de mayor prevalencia, afectando entre 3,0\% y 7,5\% de los niños en edad escolar ${ }^{1}$.

Como factores etiopatogénicos se han involucrado componentes genéticos y ambientales, que operarían en proporciones variables según cada caso. Sin embargo, el peso del componente genético parece preponderante, estimándose una heredabilidad en torno a $80 \%$, según diversos estudios comparativos de gemelos mono y dicigóticos $^{2}$.

Un conjunto de hallazgos indica que en la base del SDA se encuentra una disfunción de la neurotransmisión dopaminérgica hacia la corteza prefrontal. En efecto, estudios de resonancia nuclear magnética (RNM) y flujo sanguíneo cerebral en pacientes con SDA, revelan que lo más constante es la hipoperfusión del caudado y la corteza prefrontal y una disminución del volumen del caudado del hemisferio dominante y de regiones involucradas en funciones ejecutivas que reciben aferencias dopaminérgicas ${ }^{3}$. Como procesos cognitivos anormales en el origen del SDA se han señalado diversas funciones ejecutivas propias de la corteza prefrontal (déficit en la función de inhibición de las respuestas, aversión por el retardo, déficit en el procesamiento temporal y déficit en working memory) ${ }^{1}$. Se sabe que los axones dopaminérgicos del circuito meso-cortical y meso-límbico forman un complejo plexo en la corteza prefrontal de primates y humanos, modulando el estímulo excitatorio a las neuronas piramidales glutamatérgicas de esta región $n^{4}$. El tratamiento psicoestimulante (anfetamina y metilfenidato), se basa en la facilitación de la transmisión dopaminérgica por estimulación de la liberación de dopamina desde las vesículas presinápticas e inhibición de la recaptación de este neurotransmisor del espacio sináptico ${ }^{5}$. En base a lo anterior, en la búsqueda de determinantes hereditarios, se han investigado genes codificadores de proteínas participantes en dichos sistemas.

Aunque con controversias, alelos de al menos dos genes del sistema dopaminérgico han demostrado asociación positiva con SDA en diversos estudios: el alelo de 7 repeticiones del gen codificante para el receptor dopaminérgico tipo 4 (DRD4/7R) y el alelo de 10 repeticiones (480 pb) del transportador de dopamina tipo 1 (DAT1/10R).
Estos alelos han sido asociados a los tres rasgos del SDA: hiperactividad, impulsividad e inatención 6 .

El alelo DRD4/7R, ha sido asociado, además, al temperamento búsqueda de novedad (novelty seeking) y a la propensión a abuso de substancias y parece tener una frecuencia relativa mayor en la población de Sudamérica ${ }^{6}$. En un trabajo reciente de nuestro grupo, se compararon frecuencias de presentación de distintos alelos DRD4 encontrados en población mixta chilena con datos publicados para poblaciones europeas e indígenas sudamericanas. Se obtuvo una frecuencia alélica de 0,27 para DRD4/7R, valor situado entre aquel exhibido por poblaciones europeas, que se aproxima al 0,13 y aquel presentado por algunas poblaciones nativas del cono sur de América. Por otra parte, el alelo DAT1/10R mostró tener una frecuencia alta en la población mixta chilena $(0,74)$. Las poblaciones indígenas sudaméricanas se caracterizan por presentar valores aún más altos, mientras que las europeas exhiben frecuencias más bajas? ${ }^{7}$.

Los pacientes con SDA y alelo DRD4/7R positivo no mostrarían defecto atencional significativo, pero sí las otras facetas conductuales (hiperactividad e impulsividad). Este se correlacionaría mejor con ciertas dimensiones de la personalidad (extraversión) o del temperamento (novelty seeking). Estos niños se aburren rápidamente en ausencia de condiciones altamente estimulantes y muestran intolerancia por la espera, con un estilo diferente, que puede ser adaptativo en ciertas circunstancias. Por otra parte, pacientes portadores de SDA DRD4/7R (-) serían un grupo heterogéneo que se correlacionaría con otros factores (diferentes alelos de DRD4 o de DAT1 y lesión cerebral mínima, entre otras) ${ }^{8-10,11}$.

En este trabajo investigamos la presencia de los alelos del receptor DRD4 y del transportador de dopamina DAT1 en pacientes diagnosticados con SDA pertenecientes a familias de la Región Metropolitana de Chile.

\section{MATERIAL Y MÉTODO}

Se estudiaron 26 individuos de ambos sexos, con edades entre 9 y 14 años. Su evaluación clínica evidenció niveles de inteligencia normales, diag- 
nóstico de SDA (tipo combinado) y tratamiento efectivo con psicoestimulantes. Además, se evaluaron cuidadosamente los contextos familiar y escolar, antecedentes mórbidos perinatales y desarrollo psicomotor. Se construyeron genealogías familiares, abarcando a lo menos dos generaciones. Fueron referidos para estudio desde los centros asistenciales Hospital Calvo Mackenna, Hospital San Borja Arriarán y Clínica Las Condes, donde son controlados regularmente.

Previo a su inclusión en este estudio, los padres de estos individuos firmaron un formulario de consentimiento informado, donde se especifica claramente la confidencialidad de los datos obtenidos y su utilización estricta en investigación clínica. El protocolo de esta investigación fue aprobado por el Comité de Ética de la Facultad de Medicina de la Universidad de Chile.

En los individuos a estudiar, se certificó el cumplimiento de los criterios de tipificación de Síndrome de Déficit Atencional con Hiperactividad tipo combinado, según DSM-IV en su sección Trastornos por déficit de atención y comportamiento perturbador ${ }^{9}$. Con el objetivo de estudiar las pautas de comportamiento social y familiar de los individuos incluidos en este estudio, se aplicó el instrumento test de Conners abreviado a los padres, evaluando preferentemente la conducta de los hermanos no afectados por la psicopatología. Posteriormente, todos los pacientes fueron sometidos a la batería de evaluación de inteligencia de Wechsler revisada (WISC-R) para niños, objetivando su coeficiente intelectual y obteniendo una apreciación cualitativa de su conducta.

Con el objeto de indagar sobre la existencia de asociación entre SDA y los alelos DRD4/7R y DAT1/10R se compararon los niños con diagnóstico de SDA (casos) con sus hermanos sin diagnóstico de SDA (controles). Este método, que denominaremos de casos y controles con base familiar (CCBF), tiene ventajas sobre el método tradicional de análisis de asociación poblacional, porque disminuye o evita factores de sesgo como la estratificación poblacional, debido a que tanto el grupo de casos como el de controles poseen composiciones genéticas semejantes por pertenecer a las mismas familias.

Existen otros métodos para evitar la estratificación poblacional como el AFBAC (affected family based controls) ${ }^{12}$, el TDT (transmission test for linkage disequilibrium) ${ }^{13}$ y el ETDT (extended transmission disequilibrium test) ${ }^{14}$. Nuestra información genealógica es insuficiente para aplicar estos métodos más complejos que requieren mayor número de observaciones. En efecto, aún no hemos podido obtener la colaboración de numerosos padres para la obtención de especímenes de sangre.

Hemos subdividido a los pacientes en categorías cuando, en base a la recopilación de antecedentes anamnésticos, el SDA aparece presente en otros miembros de la familia (agregación familiar) y no hay factores en la historia que sugieran un SDA secundario, como por ejemplo tabaquismo materno durante el embarazo, patologías del embarazo y patologías perinatales entre otros.

El estudio de tipificación genético molecular incluyó la toma de una alícuota de sangre periférica por punción venosa estandarizada, el aislamiento de linfocitos y la posterior extracción del ADN genómico de cada paciente. Luego se utilizó la técnica de reacción en cadena de la polimerasa (PCR) para la amplificación de regiones polimórficas conteniendo los VNTR (variable number of tandem repeats) de los genes para el receptor de dopamina D4 y el transportador DAT1. Este procedimiento se llevó a cabo utilizando básicamente el protocolo descrito por Nanko et al $(1993)^{15}$. La genotipificación de cada paciente se realizó en forma directa, según la visualización en un gel de agarosa 2,5\% teñido con bromuro de etidio de los productos de la amplificación anteriormente señalados.

\section{RESULTADOS}

De 26 sujetos analizados, 24 correspondieron a individuos de sexo masculino y 2 a sexo femenino. Según anamnesis realizada a los pacientes, se subdividió el grupo en 16 individuos con historia sugerente de causa genética, 3 con historia sugerente de causa ambiental, 4 con historia inespecífica y 3 individuos que no pudieron ser asignados a alguna de las categorías anteriores por la falta de datos concluyentes.

$\mathrm{Al}$ verificar la presencia del alelo DRD4/7R en los casos estudiados con diagnóstico de SDA (13), se definieron 11 casos de SDA probablemente genético, 1 caso de etiología probablemente am- 
Tabla 1. Comparación de frecuencias genotípicas absolutas para los alelos D RD 4/7R y D AT 1/10R en niños sanos y afectados de SD A pertenecientes a familias de la Región M etropolitana

\begin{tabular}{|lccc|ccc|c|}
\hline $\begin{array}{c}\text { Homocigotos } \\
\text { DRD4/7R }\end{array}$ & $\begin{array}{c}\text { Heterocigotos } \\
\text { DRD4/7R }\end{array}$ & $\begin{array}{c}\text { Otros } \\
\text { genotipos }\end{array}$ & $\begin{array}{c}\text { Homocigotos } \\
\text { DAT1/10R }\end{array}$ & $\begin{array}{c}\text { Heterocigotos } \\
\text { DAT1/10R }\end{array}$ & $\begin{array}{c}\text { Otros } \\
\text { genotipos }\end{array}$ & Totales \\
\hline Casos & - & 13 & 13 & 16 & 10 & - & 26 \\
Controles & 6 & 19 & 10 & 15 & - & 25 \\
\hline
\end{tabular}

biental y 1 caso de etiología inespecífica. La ausencia del alelo DRD4/7R (13) se verificó en 3 casos de etiología probablemente genética, 2 casos probablemente ambientales y 8 casos de etiología inespecífica o discordante. Cabe destacar que todos los sujetos de estudio en los cuales se detectó la presencia del alelo, presentaban éste al estado heterocigoto. Debido a que el alelo DAT1/ $10 \mathrm{R}$ tiene mayor frecuencia génica, éste estaba presente en todos los individuos al estado homocigoto $(0,51)$ o heterocigoto $(0,49)$.

En la Tabla 1 se señalan los resultados de la comparación de frecuencias genotípicas absolutas de DRD4/7R y DAT1/10R en niños sanos y afectados.

Cabe señalar que, al comparar las frecuencias genotípicas de DRD4/7R (heterocigotos) en niños con y sin diagnóstico de SDA, se observó una mayor frecuencia de este genotipo en los primeros, siendo la diferencia estadísticamente significativa (prueba exacta de Fisher, $p=0,05)^{16}$. Al comparar frecuencias similares en padres positivos para SDA por anamnesis con los equivalentes sanos, las diferencias tuvieron una tendencia similar, pero sin alcanzar significación estadística. La frecuencia génica del alelo $\mathrm{DRD} 4 / 7 \mathrm{R}$ en las familias estudiadas fue de 0,19, inferior a las frecuencias descritas por nosotros para el área norte de Santiago ${ }^{7}$, indicando un origen más europeo. En efecto, los niños con SDA tuvieron una frecuencia de $\mathrm{DRD} 4 / 7 \mathrm{R}$ de 0,25 , en cambio los niños no afectados exhibieron frecuencias características de poblaciones europeas $(0,12)$.

Por otra parte, al comparar las frecuencias genotípicas de DAT1/10R entre casos y controles estos últimos presentaron valores más bajos, pero las diferencias no fueron significativas (prueba exacta de Fisher $\mathrm{p}=0,10$ ). Las frecuencias génicas de DAT1/10R fueron de 0,81 en casos y 0,70 en controles.

\section{DisCUSIÓN}

En el presente trabajo informamos de resultados sobre la frecuencia del alelo DRD4/7R en una muestra de pacientes en tratamiento por SDA. Se trata del primer estudio nacional acerca de la presencia de esta variante genética en individuos con diagnóstico de SDA.

Empleando el método CCBF, encontramos que la frecuencia del alelo 7R es mayor en la población de niños y adolescentes portadores de SDA, comparada con la frecuencia observada en el conjunto de hermanos sin SDA. Esta diferencia alcanza significación estadística. Observamos, también, una mayor frecuencia de presentación de este alelo cuando existe la sospecha clínica de que el SDA sea un rasgo heredado, en base a que se reporta en la anamnesis agregación familiar.

Cabe además mencionar que, la frecuencia del alelo 7R tiende a ser mayor en los padres de los niños estudiados cuando éstos tienen diagnóstico de SDA por anamnesis. Sin embargo, esta asociación no alcanza significación estadística, posiblemente debido a la inexactitud del diagnóstico.

No observamos una asociación DRD4/SDA completa, es decir, se presentan casos en que, por la historia, el rasgo SDA aparece como una condición familiar heredada, sin embargo, no se observa la variante $7 \mathrm{R} \mathrm{y}$, a la inversa, encontramos también el alelo de riesgo en individuos sin clínica de SDA.

Un importante factor de sesgo en los estudios poblacionales de asociación, es la estratificación poblacional, que determina que un gen marcador aparece asociado a una determinada patología, debido a que casos y controles pertenecen a poblaciones con composiciones genéticas contrastantes. En Latinoamérica, este factor de sesgo es muy común cuando se eligen casos y controles 
pertenecientes a diferentes niveles socioeconómicos, ya que éstos difieren en su composición genética, exhibiendo los niveles más bajos, un porcentaje mayor de genes amerindios.

Las familias estudiadas por nosotros son de clase media, no tienen apellidos indígenas y presentan frecuencias génicas de DRD4/7R más europeas que la población de dadores de sangre de los hospitales José Joaquín Aguirre y Roberto del Río. De hecho, los niños sin SDA presentan frecuencias típicamente europeas $(0,12)$ y sus hermanos diagnosticados con SDA presentan, como es de esperar en caso de asociación positiva, una frecuencia más alta $(0,25)$.

Desde hace años se concibe al SDA como una condición que obedece a múltiples causas, genéticas y ambientales. Puede ser descrito entonces como un rasgo cuantitativo de dependencia multifactorial. Esto implica que un factor aislado no sería suficiente para causar el síndrome, pero que la suma de varios factores de riesgo originaría su aparición. Los descubrimientos recientes de la genética humana están influyendo en la concepción de que el SDA, más que una patología, sería un rasgo temperamental heredado (un fenotipo conductual), de allí que se observe en varios individuos de una misma familia. En consecuencia, es probable que se requiera, además de la presencia de $\mathrm{DRD} 4 / 7 \mathrm{R}$, de varios genes de susceptibilidad más la presencia de factores ambientales de riesgo para que éste se exprese.

\section{REFERENCIAS}

1. Casteldanos FX, Tannok R. Neuroscience of attention-deficit/hyperactivity disorder: the search for endophenotypes. Nat Rev Neurosci 2002; 3: 61728.

2. Thapar A, Holmes J, Poulton K, Harrington R. Genetic basis of attention deficit and hyperactivity. Br J Psychiatry 1999; 174: 105-11.

3. Casteluanos FX, Lee PP, Sharp W, Jefrries NO, Greenstein DK, Clasen LS et aL. Developmental trajectories of brain volume abnormalities in children and adolescents with attention-deficit/ hyperactivity disorder. JAMA 2002; 288: 1740-8.

4. Krimer L, JaKab R, Goldman-RaKic P. Quantitative three-dimensional analysis of the catecholaminer-
El mecanismo postulado que vincula al alelo DRD4/7R con el SDA guarda relación con el hecho que la variación alélica de 7 repeticiones se produce en el exón 3 del gen, zona codificante del segmento proteico del receptor que interacciona con una proteína-G, cuya activación es la fase inicial de la cascada de transducción de señales gatillada por la interacción dopamina-receptor. En síntesis, si un sujeto tiene la variante $\mathrm{DRD} 4 / 7 \mathrm{R}$, se daría una condición en que el circuito mesocortical dopaminérgico sería menos eficiente en facilitar la activación de la corteza prefrontal, lo que a su vez se traduciría en una deficiencia en las funciones ejecutivas que fallan en el SDA. Existe, sin embargo, una infinidad de otras proteínas, también participantes en los sistemas cerebrales mencionados, que no han sido analizadas.

En el futuro pretendemos incrementar el número de genes a analizar, en general en la línea de aquellos codificantes de proteínas de sistemas de neurotransmisores. Estos estudios forman parte de un conjunto de pruebas imagenológicas, electrofisiológicas y cognitivas que estamos desarrollando en niños y adolescentes portadores de SDA y también en sus padres. Pretendemos optimizar, con el tiempo, el diagnóstico de SDA en los adultos, siendo el propósito último profundizar en la comprensión de esta condición con miras a poder diagnosticarla en forma precoz y enfrentarla más apropiadamente en términos educacionales y terapéuticos.

gic innervation of identified neurons in the macaque prefrontal cortex. J Neurosci 1997; 17: 7450-61.

5. Kandel E, Schwartz J, Jessel T. Essentials of Neural Science and Behavior. Appleton and Lange. Simon and Schuster, 1995.

6. La Hoste G, Swanson J, Wigal S, Giabe C, Wigal T, KING N ET AL. Dopamine D4 receptor gene polymorphism is associated with attention deficit hyperactivity disorder. Mol Psychiatry 1996; 1: 121-4.

7. Vieyra G, Moraga M, Henríquez H, Aboitiz F, RothHAMmER F. Distribución de alelos de los genes DRD4 y DAT1 del sistema dopaminérgico en la población mixta de Santiago de Chile. Rev Méd Chile 2003; 131: 135-43. 
8. Swanson J, Sunohara G, Kennedy J, Regino R, Fineberg E, Wigal T ET aL. Association of the dopamine receptor D4 (DRD4) gene with a refined phenotype of attention deficit hyperactivity disorder (ADHD): a family-based approach. Mol Psychiatry 1998; 3: 38-41.

9. Pichot P, López-Ibor J, Mirar M. Manual Diagnóstico y estadístico de los transtornos mentales, DSM IV. Ed. Masson S.A., 1995.

10. Swanson J, Oosterlaan J, Murias M, Schuck S, Flodman P, Spence M et al. Attention deficit/ hyperactivity disorder children with a 7-repeat allele of the dopamine receptor D4 gene have extreme behavior but normal performance on critical neuropsychological tests of attention. PNAS 2000; 97: 4754-9.

11. Ding Y-CH, Chi H-C, Grady D, Morishima A, Kidd J, KIDD K ET AL. Evidence of positive selection acting at the human dopamine receptor D4 gene locus. PNAS 2002; 99: 309-14.

12. Thомsоn G. HLA disease associations: models for insulin dependent diabetes mellitus and the study of complex human genetic disorders. Annu Rev Genet 1988; 22: 31-50.

13. Spielman RS, McGinnis RE, Ewens WJ. Transmission test for linkage disequilibrium: the insulin gene region and insulin-dependent diabetes mellitus (IDDM). Am J Hum Genet 1993; 52: 506-16.

14. Shan P. Association between a complex disorder and a marker. In: Statistics in Human Genetics. N. Demis (ed) London. Arnold Publishers 1999; 16183.

15. Nanko S, Hattori M, Ikeda K, Ssaki T, Kazamatsuri H, Kuwata S. Dopamine D4 receptor polymorphism and schizophrenia. Lancet 1993; 341: 68990.

16. Dixon WJ, Massey FJ. Introduction to Statistical Analysis. Mc Graw Book Company. New York, 1969.

\section{Agradecimientos}

Este trabajo fue parcialmente financiado a través de fondos otorgados por el Proyecto Fondecyt \# 1010816, el Millenium Nucleus for Integrative Neuroscience y el Instituto Milenio CBB. Agradecemos muy sinceramente a los Drs. Freya Fernández K., y Ricardo García S., por su importante participación en el reclutamiento de pacientes, a los niños y padres por su desinteresada colaboración y a un revisor anónimo por sugerencias que contribuyeron a mejorar el manuscrito sustancialmente. 